\title{
Prevalence and management of severe asthma in primary care: an observational cohort study in Sweden (PACEHR)
}

\author{
Kjell Larsson ${ }^{1 *}$, Björn Ställberg ${ }^{2}$, Karin Lisspers², Gunilla Telg ${ }^{3}$, Gunnar Johansson², Marcus Thuresson ${ }^{4}$ \\ and Christer Janson ${ }^{5}$
}

\begin{abstract}
Background: Severe and uncontrolled asthma is associated with increased risk of exacerbations and death. A substantial proportion of asthma patients have poor asthma control, and a concurrent COPD diagnosis often increases disease burden. The objective of the study was to describe the prevalence and managemant of severe asthma in a Swedish asthma popuöation.

Methods: In this observational cohort study, primary care medical records data (2006-2013) from 36 primary health care centers were linked to data from national mandatory Swedish health registries. The studied population (>18 years) had a record of drug collection for obstructive pulmonary disease (ATC code R03) during 2011-2012, and a physician diagnosed asthma (ICD-10 code J45-J46) prior to drug collection. Severe asthma was classified as collection of high dose inhaled steroid (> 800 budesonide or equivalent per day) and leukotriene receptor antagonist and/or long-acting beta-agonist. Poor asthma control was defined as either collection of $\geq 600$ doses of short-acting beta-agonists, and/or $\geq 1$ exacerbation(s) during the year post index date.
\end{abstract}

Results: A total of 18,724 asthma patients (mean 49 years, $62.8 \%$ women) were included, of whom 17,934 (95.8\%) had mild to moderate and 790 (4.2\%) had severe asthma. Exacerbations were more prevalent in severe asthma (2.59 [2.41-2. 79], Relative Risk [95\% confidence interval]; $p<0.001$ ). Poor asthma control was observed for $28.2 \%$ of the patients with mild to moderate asthma and for more than half $(53.6 \%)$ of the patients with severe asthma $(<0.001)$. Prior to index, one in five severe asthma patients had had a contact with secondary care and one third with primary care. A concurrent COPD diagnosis increased disease burden.

Conclusion: Severe asthma was found in $4.2 \%$ of asthma patients in Sweden, more than half of them had poor asthma control, and most patients had no regular health care contacts.

Keywords: Severe asthma, Asthma prevalence, Uncontrolled asthma

\section{Background}

Asthma treatment has improved during the last 3 decades and mortality because of to asthma has decreased [1]. Notwithstanding, many asthma patients lack full asthma control as defined by international guidelines [2]. In a survey of nearly 3500 patients with asthma, three of four patients inhaled short-acting beta-agonists (SABA) on a daily basis and half of the patients had uncontrolled

\footnotetext{
* Correspondence: kjell.larsson@ki.se

${ }^{1}$ Work Environment Toxicology, The National Institute of Environmental

Medicine, Karolinska Institutet, Stockholm, Sweden

Full list of author information is available at the end of the article
}

asthma [3]. In another study almost half of the asthma patients experienced day time symptoms, one third had sleep disturbances, and one of four reported unscheduled emergency/health care visits [4]. Still, half of the patients who reported severe persistent symptoms of asthma considered their asthma to have been well or completely controlled indicating that individuals with asthma becomes accustomed to their symptoms [4].

Severe asthma is associated with high risk of severe exacerbations and death [5], and represents a high proportion of the total costs for asthma care [6, 7]. There is no uniform definition of severe asthma, but according to 
ERS/ATS and GINA guidelines severe asthma is defined as asthma that requires treatment with high doses of inhaled corticosteroids plus a second controller, and/or systemic corticosteroids, to prevent it from becoming uncontrolled, or remains uncontrolled despite this therapy $[2,8]$. Swedish recommendations on asthma management are similar to the GINA guidelines [2], in which it is recommended that severe asthma should be managed by respiratory/allergy specialists $[2,5,9]$. Most patients with asthma have a mild or moderate disease and data on the prevalence of severe asthma is sparse but reports indicate between 5 and $10 \%$ of the asthmatic populations to have had severe disease $[8,10]$. In a recent observational study, $8.1 \%$ were reported to have severe asthma [11]. In that study, severe asthma was defined as a need for a high dosage of inhaled steroids added to a second controller (long-acting beta-agonists (LABA), leukotriene modifiers (LTRA), theophylline or treatment with omalizumab [11]).

Uncontrolled asthma is not always synonymous with severe disease. Poor control may depend on refractoriness to treatment, but may also be caused by other factors such as poor treatment adherence, which may be due to costs, side effects, inability to understand how to use inhalers or negligence [12]. Indicators of poor asthma control are high consumption of rescue medication (SABA), need for repeated oral steroid treatments, visits to the emergency department, and hospitalizations because of asthma [11].

In Sweden, the overall asthma prevalence is approximately $8 \%$ [13]. The present observational study focuses on the prevalence of severe and mild to moderate asthma in Swedish primary care and the characterization of these patients regarding asthma control, management and co-morbidities.

\section{Methods}

\section{Study design}

This observational cohort study linked primary care medical records data to data from national mandatory Swedish health registries.

All asthma patients were identified at 36 primary care centers. Electronic medical records data (e.g. date of birth, sex, diagnoses by ICD-10-CM codes, primary healthcare center contacts, lung function assessments and drug prescriptions) was extracted from 2006 to 2013 using an established software system (Pygargus Customized eXtraction0, Program, $\mathrm{CXP}^{\mathrm{m}}$ ) [14]. Data were also extracted from Swedish national health registries, covering mandatory individual health data on a full population level. Data regarding morbidity and mortality were collected from the National Patient Register, inpatient hospital care (admission and discharge dates, main and secondary diagnoses specified by ICD-10-CM code) and outpatient hospital care (number of contacts, diagnoses), and the Cause of Death Register (date and cause[s] of death), respectively. Data on drug prescriptions from hospital and primary care were collected from the Swedish Prescribed Drug Register. The personal identification number used to identify included patients was replaced with a study ID number prior to further data processing. The Swedish National Board of Health and Welfare performed data linkage. The Department of Medical Sciences, Respiratory Medicine at Uppsala University, Sweden, managed the linked database. The study protocol was approved by the regional ethics committee in Uppsala, Sweden (reference number 2014/446).

\section{Study population}

The population included males and females $\geq 18$ years of age with a record of drug collection for obstructive pulmonary diseases (Anatomical Therapeutic Chemical (ATC) code R03) during 2011-2012, and a physiciandiagnosed asthma (ICD-10 code J45-J46) established prior to drug collection. Patients with a diagnosis of polymyalgia rheumatica (ICD-10 code M35.3) or rheumatoid arthritis (ICD-10 code M05) were excluded minimizing the number of patients using oral corticosteroid for other causes than asthma. Index date was defined as first collection of an R03 drug during 2011-2012 and the patients were followed 1 year post index date.

Classification of asthma severity was based on the R03 collection at index date. Severe asthma was classified as collection of high dosage inhaled steroid (ICS, $>800$ budesonide or equivalent per day) and LTRA and/or LABA collected at the same time or within a 3 months' period before or after the ICS. Patients who did not fulfil the severe asthma criteria were classified as having mild to moderate asthma.

\section{Outcomes and variables}

Asthma exacerbations were defined as asthma-related hospitalizations (ICD-10 code J45 or J46 as primary diagnosis), hospital emergency visits because of asthma, and/ or collection of oral steroids (ATC code H02AB). Exacerbations occurring within a 14 days' period were calculated as one event. Poor asthma control was defined as either collection of more or equal to 600 doses of SABA, and/or $\geq 1$ exacerbations during the year post index date.

Visits in primary care and outpatient specialist care (to physician or nurse) was defined by a recorded asthma diagnosis in the medical record or registry (ICD-10 code J45 or J46). Mortality was registered post index date to December 31, 2013, i e a follow up duration of 1-2 years.

\section{Statistical analysis}

Statistical analyses were performed using SAS version 9.3 and $\mathrm{R}$ version 3.2.3. Baseline characteristics are described as mean (standard deviation, SD) for continuous 
variables and absolute and relative frequencies for categorical variables. Crude and age adjusted data were calculated. Because of differences in age distribution between groups, the baseline tables were age-standardized using a direct age standardization (i e all observations were weighted relative to the proportion of patient in the specific age group in the total population). Comparisons of baseline characteristics of the age-adjusted values were performed using Chi-square tests. Comparisons of the two asthma severity groups regarding rates of exacerbations and asthma control for the year after index were performed using a Poisson regression, adjusted for age.

\section{Results}

A total of 18,724 adult patients with asthma were included in this cohort. The mean age was 49.1 years, $62.8 \%$ were women, and post-bronchodilator $\mathrm{FEV}_{1}$ was $82.6 \%$ of predicted value. Severe asthma was identified in 790 patients (4.2\%), and 17,934 patients had mild to moderate asthma (Table 1A, Fig. 1). As a control pf consistency it was found that $96 \%$ of the patients with severe asthma collected high doses of inhaled steroids also the year after index date. Patients with severe asthma were older at index date $(p<$ $0.001)$ and at asthma diagnosis $(p<0.001)$, and had lower $\mathrm{FEV}_{1}$ and FVC than patients with mild to moderate asthma $(p<0.001$, Table 1A).

\section{The year prior to index date}

During the year prior to index date, 100 exacerbations/ 100-patient-years were observed for severe asthma and 34/100-patient-years for mild to moderate asthma
(Relative Risk, RR [95\% confidence interval] 2.59 [2.412.79]; $p<0.001)$. Hospitalizations because of asthma were 3.0/100-patient-years for severe asthma and 0.6/ 100-patient-years for mild to moderate asthma (RR 4.84 [3.09-7.57]; $p<0.001)$. Mean number of oral steroid claims were 97/100-patient-years for severe asthma and 33/100-patients years for mild to moderate asthma (RR 2.57 [2.38-2.77]; $\mathrm{p}<0.001$ ). No difference between the groups was found regarding emergency visits at hospital because of asthma exacerbations (RR 0.67 [0.09-4.93]; $p=0.694)$.

In the severe asthma group, one-fifth of the patients had visited secondary care, and one third had visited primary care because of asthma. Visits because of asthma were more frequent in severe asthma than in mild to moderate asthma, while total visits (all causes) to primary care did not differ between the groups (Table 2A).

During the year prior to index date, $88.1 \%$ in the severe and $65.2 \%$ in the mild to moderate asthma group collected any R03 medication $(p<0.001$, Table 3A). Short-acting beta-agonists (SABA) were collected by half of the patients with severe asthma and for one-third of the patients with mild to moderate disease $(p<0.001$, Table $3 \mathrm{~A}$ ). Inhaled steroids (mono or fixed combination) were collected by $85.4 \%$ with severe asthma and by $55.6 \%$ with mild to moderate asthma ( $\mathrm{p}<0.001$, Table 3A). Inhaled steroids were used in combination with LABA in $29.8 \%$ of patients with mild to moderate asthma and in $60.4 \%$ of those with severe disease while the combination of ICS and leukotriene modifiers

Table 1 Basal characteristics one year prior to or at index date of (A) all patients with mild to moderate and severe asthma and (B) after exclusion of patients with a COPD diagnosis (J44). Study population and sex are given as number and proportions (\%) of patients and other data are given as mean values (standard deviation, SD). P-values indicate differences between mild to moderate and severe asthma in each group, respectively

\begin{tabular}{|c|c|c|c|c|c|c|}
\hline & $\mathrm{A}$ & & & $\mathrm{B}$ & & \\
\hline & All patients $(n=18$, & & & Asthma only (no C & $(n=16,703)$ & \\
\hline & Mild to moderate & Severe & $P$ value & Mild to moderate & Severe & $P$ value \\
\hline Study population, n (\%) & $17,934(95.8)$ & $790(4.2)$ & $<0.001$ & $16,142(96.6)$ & $561(3,4)$ & $<0.001$ \\
\hline Female, n (\%) & $11,293(63.0)$ & $472(59.7)$ & 0.071 & $10,157(62.9)$ & $337(60.1)$ & 0.169 \\
\hline Age at index & $48.8(19.2)$ & $56.9(16.9)$ & $<0.001$ & $46.6(18.6)$ & $52.3(16.8)$ & $<0.001$ \\
\hline Age at death (years) & $78.0(13.2)$ & $81.0(8.6)$ & 0.042 & $77.2(15.1)$ & $81.0(10.2)$ & 0.165 \\
\hline BMI $\left(\mathrm{kg} / \mathrm{m}^{2}\right)^{\mathrm{a}}$ & $27.6(6.0)$ & $27.9(5.8)$ & 0.274 & $27.6(6.0)$ & $28.0(5.6)$ & 0.248 \\
\hline $\begin{array}{l}\mathrm{FEV}_{1} \% \text { predicted value post } \\
\text { bronchodilation }^{\mathrm{b}}\end{array}$ & $83.6(21.1)$ & $67.5(25.9)$ & $<0.001$ & 89.7 (15.9) & $80.0(22.1)$ & 0.003 \\
\hline $\begin{array}{l}\text { FVC \% predicted value post } \\
\text { bronchodilation }^{\text {b }}\end{array}$ & $94.7(18.6)$ & $84.3(21.1)$ & $<0.001$ & $97.7(16.5)$ & $91.1(19.8)$ & 0.043 \\
\hline $\mathrm{FEV}_{1} / \mathrm{FVC}^{\mathrm{b}}$ & $0.737(0.138)$ & $0.640(0.175)$ & $<0.001$ & $0.777(0.097)$ & $0.715(0.140)$ & 0.005 \\
\hline Eosinophils ${ }^{c}, \times 103$ cells/uL & $0.32(0.43)$ & $0.40(0.63)$ & 0.065 & $0.31(0.44)$ & $0.37(0.43)$ & 0.132 \\
\hline
\end{tabular}

${ }^{\mathrm{a}} \mathrm{BMI}$ was available in $53.0 \%$ of patients with mild to moderate asthma and $58.2 \%$ with severe asthma

${ }^{\mathrm{b}}$ Available data $(<10 \%$ of the patients in both groups) from lung function measurements performed during the year prior to index date in both groups

${ }^{c}$ Data on blood eosinophilia was available in $16.8 \%$ of patients with mild to moderate asthma and $23.8 \%$ with severe asthma 


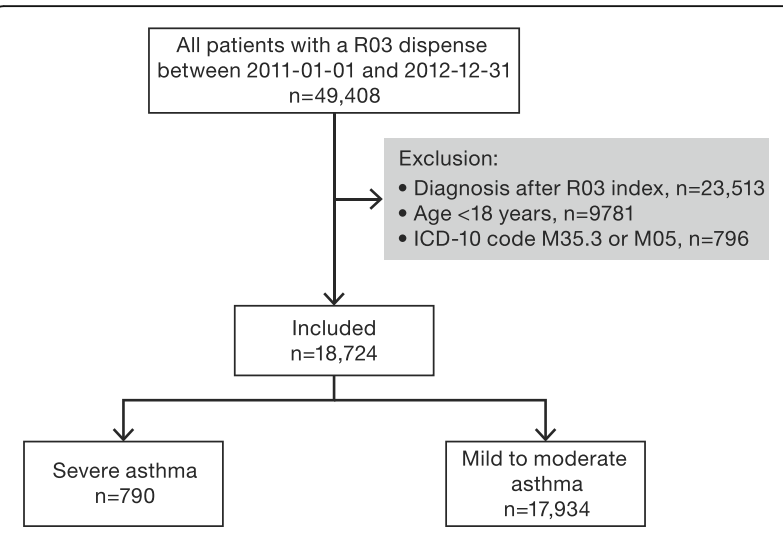

Fig. 1 Study flow chart. Out of 49,408 patients with asthma (ICD10 code J45 or J46) who, during 2011 and 2012, collected respiratory drugs (ATC R03) from the pharmacy 18,724 fulfilled the inclusion criteria, i e were above the age of 18 years, had an asthma diagnosis prior to drug collection and did not have polymyalgia rheumatic or rheumatoid arthritis. Of these 18,724 patients, 790 were classified as having severe asthma and 17,934 as having mild to moderate asthma

(montelukast) was used by $0.6 \%$ and $0.9 \%$ in mild to moderate and severe disease, respectively. Antihistamines and omalizumab as well as antibiotics alone or in combination with oral steroids were more often collected by patients with severe than for patients with mild to moderate asthma $(p<0.001$, Table 3A). Patients with severe asthma often collected hypnotics, bisphosphonates, calcium and vitamin D (Table 3A; $p \leq 0.002$ ).

During the year prior to index date, almost half of the patients in both groups experienced acute upper respiratory tract infections (Table 4A). Chronic bronchitis, nasal polyps, and influenza were more common in the severe asthma group (Table 4A). A concurrent diagnosis of chronic obstructive pulmonary disease (COPD) was found in $21.6 \%$ with severe and $10.2 \%$ with mild to moderate asthma $(p<0.001$, Table $4 \mathrm{~A})$. Pneumonia was diagnosed in $20.7 \%$ of the patients with severe asthma and in $14.4 \%$ of the patients with mild to moderate asthma $(p<0.001$; Table $4 \mathrm{~A})$. Heart failure was more common in the severe asthma group (Table 4A).

\section{The year after index date}

During 1 year after index date, poor asthma control was observed for $28.2 \%$ of the patients with mild to moderate asthma and for $53.6 \%$ of the patients with severe asthma $(<0.001$, Fig. 2$)$. In the mild to moderate and severe group, $15.2 \%$ and $28.6 \%$ of the patients, respectively $(p<0.001)$, collected more than 600 doses of SABA (Fig. 2).

Inhaled steroids, in any form, was used by $78.1 \%$ of the patients with mild to moderate asthma and $95.9 \%$ of those with severe asthma ( $p<0.001$ between the groups).

Table 2 Health care utilization one year prior to index date in (A) all patients with mild to moderate and severe asthma and (B) in patients after exclusion of the patients with a concurrent COPD diagnosis. Age adjusted data presented as number and proportion (\%) of the patients

\begin{tabular}{|c|c|c|c|c|c|c|}
\hline & \multicolumn{3}{|l|}{ A } & \multicolumn{3}{|l|}{ B } \\
\hline & \multicolumn{3}{|c|}{ Full study population } & \multicolumn{3}{|c|}{ Asthma only (no COPD) } \\
\hline & $\begin{array}{l}\text { Mild to moderate } \\
(n=17,934)\end{array}$ & $\begin{array}{l}\text { Severe } \\
(n=790)\end{array}$ & $P$ value & $\begin{array}{l}\text { Mild to moderate } \\
(n=16,142)\end{array}$ & $\begin{array}{l}\text { Severe } \\
(n=561)\end{array}$ & $P$-value \\
\hline \multicolumn{7}{|l|}{ Primary care visits (any reason) } \\
\hline Visit to the doctor & $10,922(60.9)$ & $464(58.7)$ & 0.237 & $9553(59.2)$ & 312. (55.7) & 0.100 \\
\hline Visits to the nurse & $8761(48.9)$ & $376(47.6)$ & 0.512 & $7555(46.8)$ & $248(44.1)$ & 0.242 \\
\hline Other visits & $4880(27.2)$ & $216(27.4)$ & 0.968 & $4065(25.2)$ & $135(23.7)$ & 0.458 \\
\hline \multicolumn{7}{|l|}{ Primary care visits because of asthma } \\
\hline Visit to the doctor & $3819(21.3)$ & $245(31.0)$ & $<0.001$ & $3421(21.2)$ & $179(32.0)$ & $<0.001$ \\
\hline Visits to the nurse & $749(4.2)$ & $53(6.7)$ & 0.001 & $641(4.0)$ & $36(6.5)$ & 0.005 \\
\hline Other visits & $513(2.9)$ & $37(4.7)$ & 0.004 & $380(2.4)$ & $23(4.0)$ & 0.012 \\
\hline \multicolumn{7}{|l|}{ Outpatient hospital visits } \\
\hline Any visit to specialist & $3644(20.3)$ & $296(37.5)$ & $<0.001$ & $3016(18.7)$ & $197(35.1)$ & $<0.001$ \\
\hline Visit to a specialist because of asthma & $1168(6.5)$ & $165(20.9)$ & $<0.001$ & $1068(6.6)$ & $114(20.3)$ & $<0.001$ \\
\hline \multicolumn{7}{|l|}{ Asthma related events } \\
\hline Exacerbations because of asthma & $3182(17.7)$ & $301(38.0)$ & $<0.001$ & $2620(16.2)$ & $196(35.0)$ & $<0.001$ \\
\hline Oral steroids & $3111(17.3)$ & $292(36.9)$ & $<0.001$ & $2562(15.9)$ & $190(33.8)$ & $<0.001$ \\
\hline Hospitalizations because of asthma & $93(0.5)$ & $21(2.6)$ & $<0.001$ & $72(0.4)$ & $14(2.5)$ & $<0.001$ \\
\hline $\begin{array}{l}\text { Emergency department visits because } \\
\text { of asthma }\end{array}$ & $22(0.1)$ & $2(0.3)$ & 0.620 & $20(0.1)$ & $2(0.4)$ & 0.367 \\
\hline
\end{tabular}


Table 3 Medication collected during the year prior to index date in (A) all patients with mild to moderate and severe asthma and in (B) in patients after exclusion with a concurrent COPD diagnosis. Age adjusted data presented as number (\%)

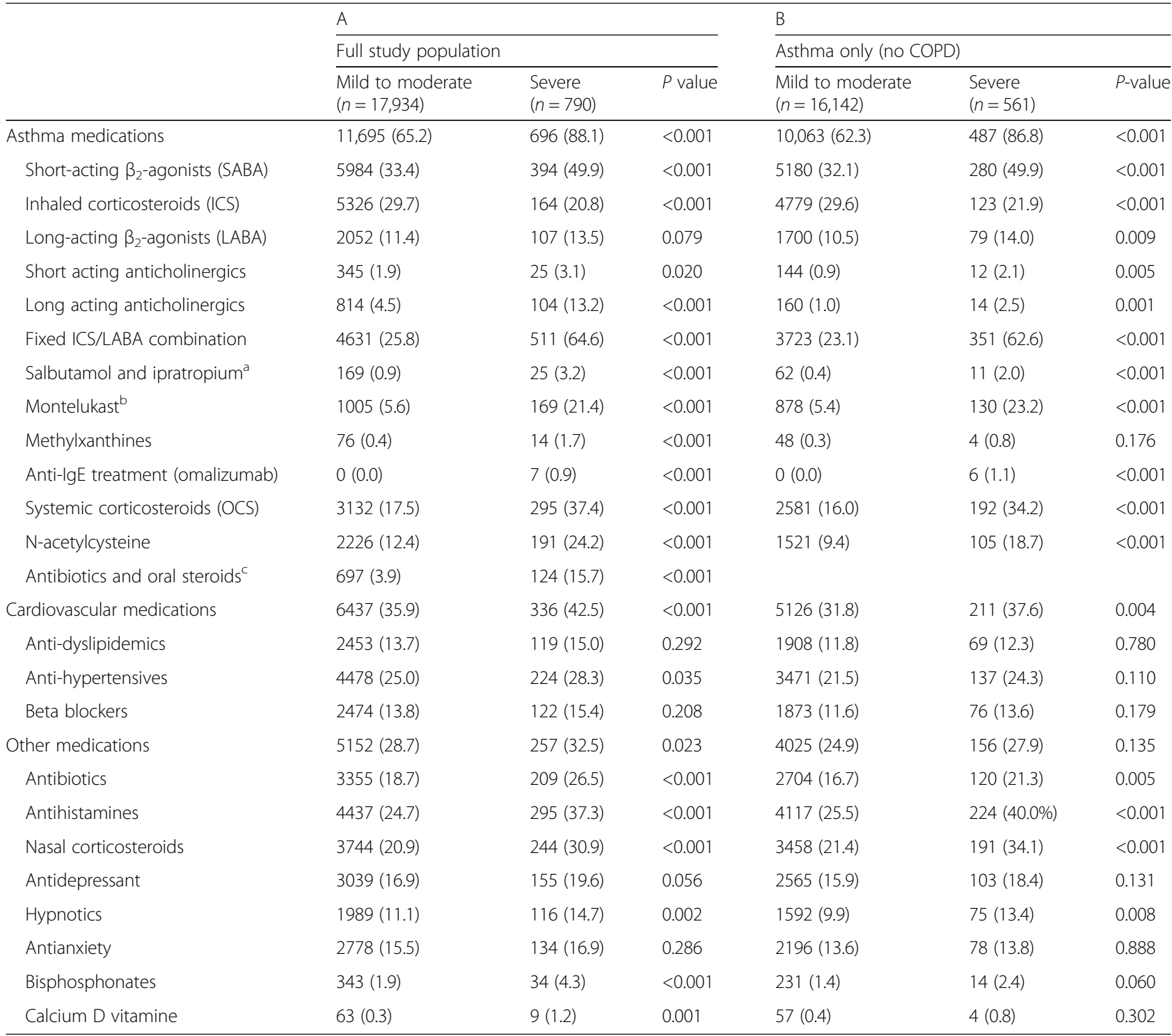

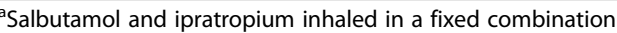

${ }^{\mathrm{b}}$ Montelukast was the only available leukotriene modifier on the market

${ }^{c}$ Antibiotics and oral steroids dispensed concomitantly

Health care utilization and exacerbations were similar the years before and after index date.

Overall, $640(3.6 \%)$ patients in the mild to moderate group and $42(5.3 \%)$ patients in the severe asthma group died during the observation period post-index. The most common causes of death were cardiovascular disease ( $33 \%$ vs $43 \%)$, neoplasms (27\% vs $17 \%$ ) and respiratory diseases (18\% vs $14 \%)$ in mild to moderate asthma and severe asthma, respectively. COPD was the most common respiratory cause of death $(81 \%$ and $83 \%$, respectively). Nine patients in the mild to moderate group ( $8 \%$ of the respiratory deaths) died from asthma, whereas no asthma deaths were observed in the severe group.

\section{Sensitivity analyses}

\section{Patients with no concurrent COPD diagnosis}

Patients with asthma only (i e no concurrent COPD diagnosis, $n=16,703$ ) were younger and had greater $\mathrm{FEV}_{1}$ and FVC than the full population (Table 1B). The difference of health care utilization remained similar between mild to moderate and severe asthma (Table $2 \mathrm{~B}$ ) whereas asthma medication (except for xanthines) was more frequently used in severe asthma (Table 3B). For patients with no concomitant COPD diagnosis longacting anticholinergics were collected for $1.6 \%$ in the mild to moderate group and for $5.2 \%$ in the severe group. Use of bisphosphonates and vitamin $\mathrm{D}$ did not differ between 
Table 4 Comorbidities during the year prior to index date in (A) all patients with mild to moderate and severe asthma and in (B) patents after exclusion of concurrent COPD diagnosis. Age adjusted data presented as number (\%)

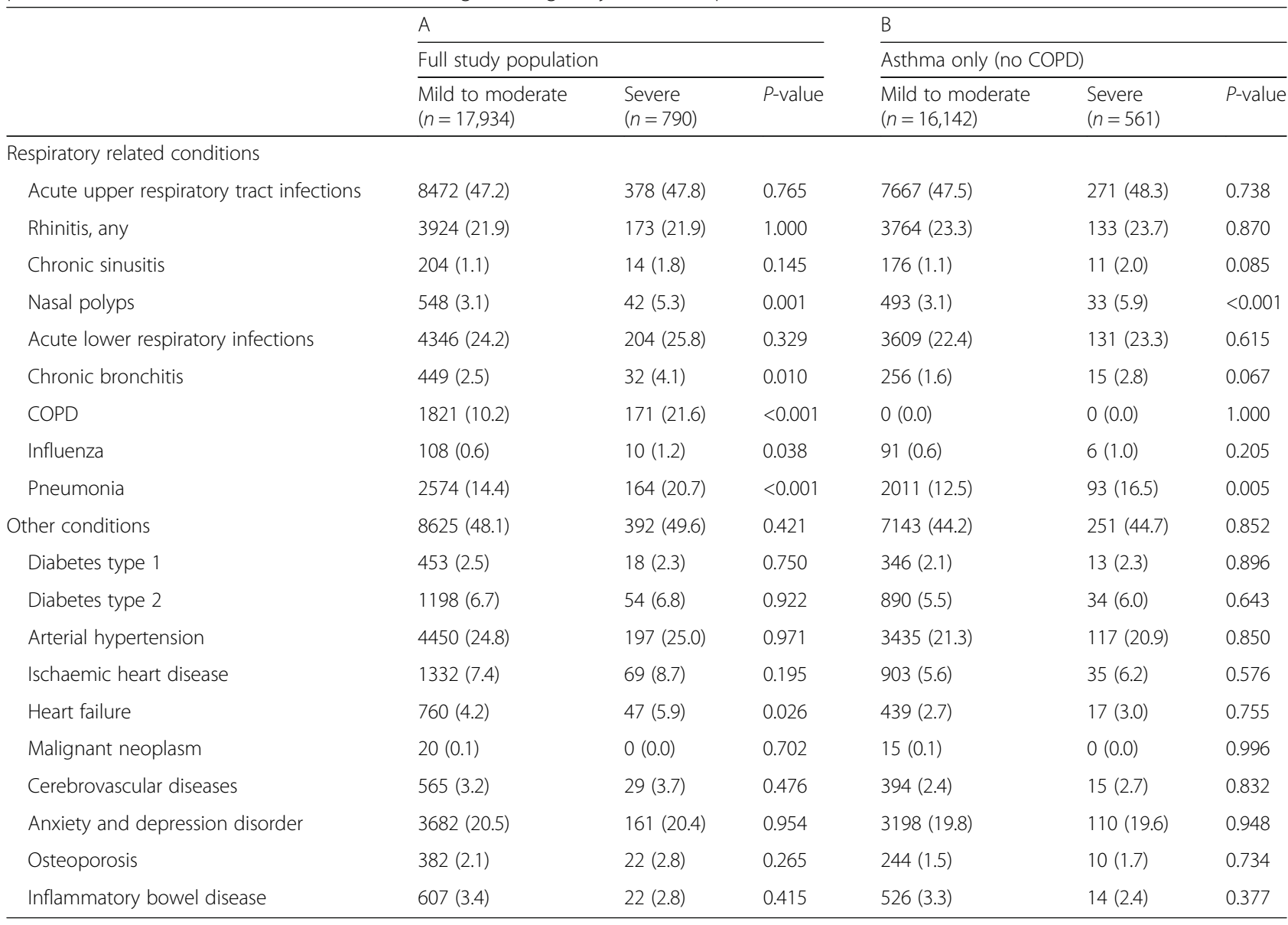

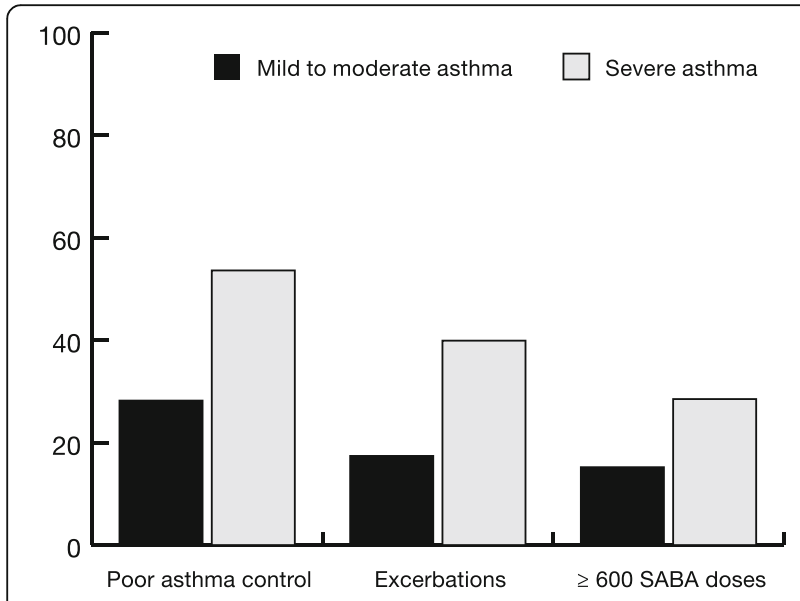

Fig. 2 Poor asthma conttrol assessed by acute exacebations and the collection of more than 600 doses of short-acting beta-2 agonists the year after index date severe and mild to moderate asthma (Table 3B). In the asthma only group, the risk of pneumonia remained greater in severe asthma whereas the prevalence of chronic bronchitis and heart failure did not differ between severe and mild to moderate asthma (Table 4B). Asthma control was better for patients with mild to moderate than for patients with severe asthma (Table 5A).

\section{Patients with both asthma and COPD diagnosis}

For patients with both asthma and COPD diagnosis $(n=$ 1902), exacerbation rate was greater for those with severe than for those with mild to moderate asthma, while hospitalization, emergency department visits and asthma control assessed as the use of SABA did not differ (Table 5B). Visits to secondary care were more frequent in severe than in mild to moderate asthma but visits to primary care did not differ (Table $5 \mathrm{~B}$ ). The use of asthma drugs was greater in the severe group, except for SABA (Table 5). For patients with both asthma and COPD diagnoses, long-acting anticholinergics was collected by $39.8 \%$ of those with mild to moderate asthma and $54.1 \%$ of those with severe asthma. In patients with 


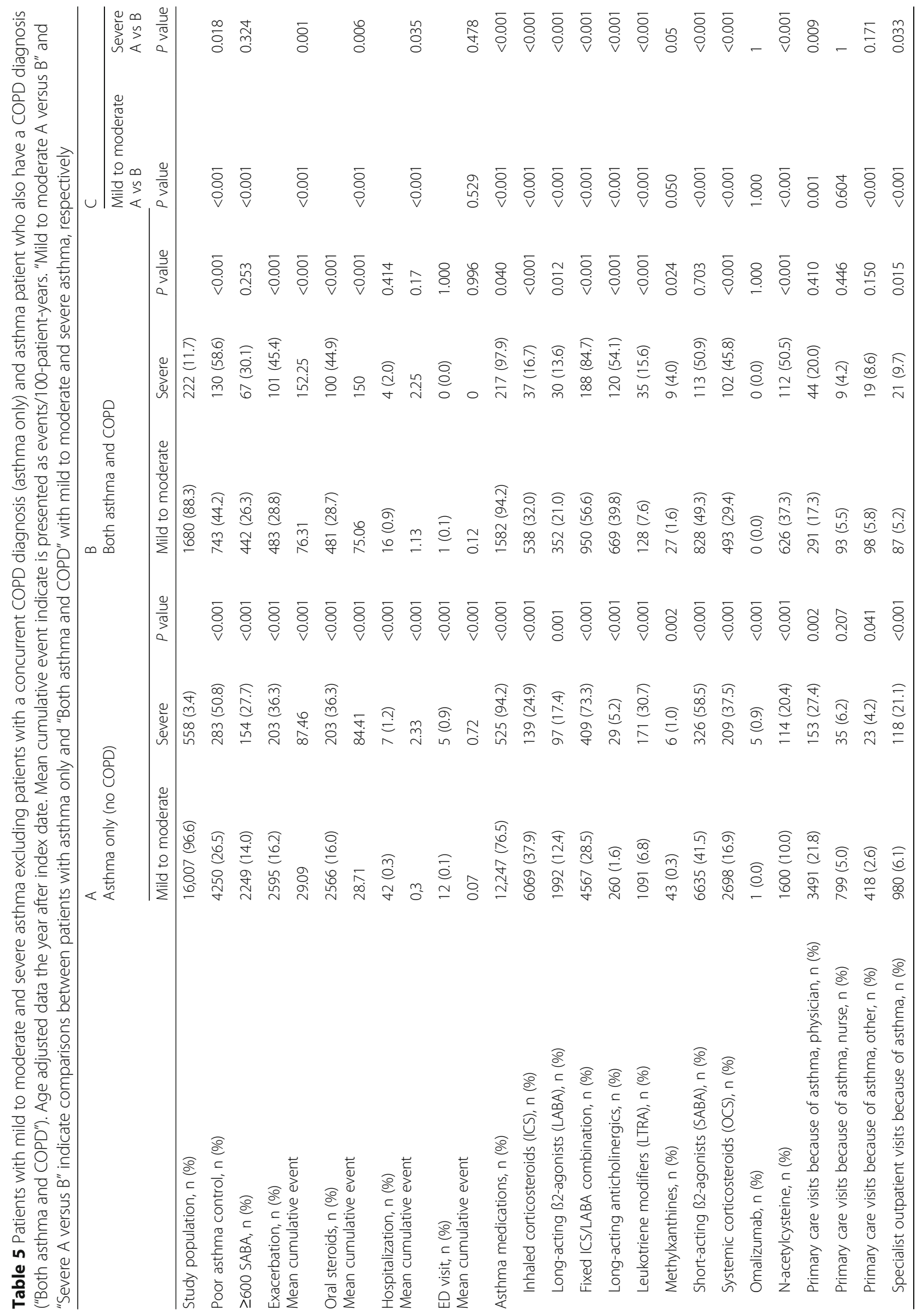


both diagnoses $31 \%$ in the mild to moderate group and $37 \%$ in the severe group had pneumonia the year prior to index date ( $p=0.084$ between groups).

\section{Asthma only patients vs patients with both asthma and COPD diagnoses}

For asthma patients with a concurrent COPD diagnosis, exacerbations rate was greater than it was for the group with asthma only irrespective of asthma severity, while asthma control assessed as use of SABA was worse in the mild to moderate group only (Table $5 \mathrm{C}$ ).

The use of asthma drugs (except for ICS as monotherapy) was more frequent in the group with both asthma and COPD diagnosis than in the asthma only group irrespective of asthma severity (Table 5C). Visits to primary care physician because of asthma and visits to secondary care were more frequent in the asthma only group than in patients with both asthma and COPD diagnosis irrespective of asthma severity, while visits to a nurse or other visits (e g physiotherapists) did not differ (Table 5C).

\section{Discussion}

In this observational cohort study, covering 18,724 asthma patients, the prevalence of severe asthma was $4.2 \%$, which is somewhat lower than the commonly reported prevalence of $5--10 \%[15,16]$. For severe asthma, the exacerbation rate was twice as high and poor asthma control twice as common as for mild to moderate asthma. In severe asthma, only one-fifth of the patients had visited secondary care and one third had a visited primary care because of asthma.

The prevalence of severe asthma was similar to a previous study, where $3.6 \%$ of patients with asthma had severe refractory asthma [17], but lower than in another study which reported a prevalence of $8.1 \%$ [11]. In that study, patients were included on a population basis, while patients were included from primary care records in our study. This may result in an underestimation of patients with severe asthma, as patients who are managed in secondary care only will not be included. In the present study, all patients above the age of 18 years were included, whereas patients above the age of 44 years were excluded in the Danish study [11]. As we did not exclude patients with a concurrent COPD diagnosis, the mean age was rather high at index date. Inclusion of older patients may have implied a "dilution" effect because of a reluctance to classify severe respiratory disease as asthma in elderly patients.

Poor asthma control (exacerbations, need for oral steroids, emergency department visits, collection of more than 600 doses of SABA during the last year) was observed for $53 \%$ of the patients with severe asthma, twice as many as in the mild to moderate asthma group. In a Danish study, with identical definition of asthma control as in our study, poor asthma control was observed for $36 \%$ of patients with severe asthma [11]. In that study, only patients below 44 years of age were included and the prevalence of severe asthma was twice as high as in the present study. These findings indicate that the older asthma population in the present study, despite similar definition of severe asthma in the two studies, had more severe disease than the younger population in the Danish study.

Exacerbation rate was twice as high, visits to primary care because of asthma were $50 \%$ greater and visits to secondary care because of asthma were three times more frequent in the severe asthma group as in the mild to moderate group. In addition, the collection of asthma medication was greater in the severe asthma group than they were for the mild to moderate group. All these differences between mild to moderate and severe asthma remained also after excluding patients with COPD clearly indicating that the high exacerbation rate, the increased utilization of health care resources and increased need for medication were mainly driven by asthma severity and not by co-existing COPD. In our study, the prevalence of COPD in the group with severe asthma was $21.6 \%$, which is similar to what has been reported by others [18].

Co-existing COPD increased the risk of exacerbations, the need for health care utilization and need for medication irrespective of asthma severity. This is in accordance with previous studies where the risk of exacerbations and hospitalization were greater in patients with features of both asthma and COPD than in patients with COPD or severe asthma [18-20]. The diagnoses were based on the physicians' judgements, indicating that patients with both diagnoses exhibited features of both asthma and COPD. Although there is likely a substantial agreement between having both an asthma and COPD diagnoses and having asthma-COPD overlap (ACO) according to GINA guidelines [2], we cannot, from the present results, assert that having both diagnosis is equal to the ACO concept. Hence, coexisting COPD increased the disease burden even more as patients with both asthma and COPD, compared with asthma only, had an increased disease burden in all these aspects unrelated to asthma severity.

As expected the asthma control was better in the mild to moderate group than in the severe group. Half of the patients with severe asthma and one of four patients with mild to moderate asthma had poor asthma control. This is in agreement with previous studies in which approximately half of patients reported daytime symptoms or were regarded as having uncontrolled asthma as assessed by the Asthma Control Questionnaire [3, 4]. 
Our data confirm previous results showing that a substantial number of patients with mild or moderate asthma have an uncontrolled disease.

Surprisingly, we found $50 \%$ greater occurrence of pneumonia in the group with severe asthma than in the mild to moderate asthma group. Treatment with ICS increases the risk of pneumonia in COPD [21] although there are intra-class differences between different steroids [22, 23]. O'Byrne et al. did not find an increased risk for pneumonia in asthma patients treated with ICS [24], whereas other results indicate an association between treatment with inhaled steroids and pneumonia also in asthma [25]. The difference in pneumonia risk was increased in severe asthma vs mild to moderate asthma after exclusion of patients with a concurrent COPD diagnosis. The risk of pneumonia was also greater in the groups with a concurrent COPD diagnosis than in patients without a COPD diagnosis. These findings suggest that asthma severity and COPD by itself are associated with increased risk of pneumonia.

According to international and Swedish recommendations, severe asthma should be managed by specialists/ physicians with extensive experience of asthma management $[2,8]$. In the present study, only one out of five patients with severe asthma visited a specialist in secondary care in the year before and after index date, and the frequency was independent of a concurrent COPD diagnosis. The attendance rate to doctors in primary care was also low. Less than $60 \%$ of the asthma patients visited primary care for any reason and close to a third of the patients visited a primary care doctor because of asthma, irrespective of disease severity. This gives reasons to believe that many of these patients are not regularly visiting a doctor or a nurse but are managed by prolongation of asthma drug prescription through telephone contacts. Such a situation is unsatisfactory, particularly for patients with severe asthma.

In the present study, somewhat less than half of the patients with mild to moderate asthma and more than $10 \%$ of the patients with severe asthma did not collect inhaled steroids the year prior to index date. The reason for this is unclear. Factors such as treatment failure due to incorrect diagnosis or poor steroid response may contribute. As it could be assumed that the majority of physicians prescribe inhaled steroids to patients with severe asthma, a likely explanation is poor adherence to prescribed treatment. We do not know to what extent the patients were adherent to treatment but we do know that approximately half of the patients with mild to moderate asthma and about $85 \%$ of the patients with severe asthma collected inhaled steroids from the pharmacy. The mean age in our study was approximately 50 years and it has been shown that adherence to treatment increases with age $[26,27]$ indicating that the adherence to therapy may have been high among those who actually did collect their medication. We also conclude that 5 to $15 \%$ of the patients with severe asthma did not collect inhaled steroids which most likely contributes to the increased costs as it is well known that poor adherence to asthma treatment is associated with increased health care costs [12]. Our data demonstrate that long-acting anticholinergics are primarily used in asthma patients who also have a COPD diagnosis and not frequently as an add on treatment in asthma patients with uncontrolled asthma. Our results also demonstrate that leukotriene modifiers (montelukast being the only drug registered in Sweden) in addition to inhaled steroids is not frequently used in the treatment of asthma in Sweden, neither in mild/moderate nor in severe asthma.

The present study is based on observational registry and medical records data implying a design that may have its limitations. Data retrieval is limited to the variables registered in the databases and medication use is based on prescription collections, which do not necessary reflect how patients actually use their medications. Furthermore, exacerbation rate may have been underestimated, as it was based on acute visits to secondary care and on collection of oral steroids and acute exacerbation managed at visits to the primary care centers were not recorded. The results validate the classification of severe asthma, by showing five times more hospitalizations in the severe asthma population compared with the mild to moderate group, supporting that this really is a severe asthma population. This is corroborated by the finding that $96 \%$ of the patients with severe asthma also collected high doses of inhaled steroids from a Swedish Pharmacies the year after index date. Important strengths of the study is the primary care setting used to identify the asthma patients, and the lack of restrictions in, for example, age, socio-economic status or healthcare insurance. This non-biased data extraction from primary care electronic medical records linked with mandatory national healthcare registers with high coverage and quality provides solid and unique data.

\section{Conclusion}

In conclusion, the prevalence of severe asthma in this Swedish primary care population was $4.2 \%$. Asthma severity increased the disease burden, which was further increased in patients with a concurrent COPD diagnosis. Patients with severe asthma had few regular contacts with both primary and specialist care, and more than half of them experienced poor asthma control.

\section{Abbreviations}

ATC: Anatomical therapeutic classification system code; Cl: Confidence interval; COPD: Chronic obstructive pulmonary disease; $\mathrm{FEV}_{1}$ : Forced expiratory volume in one second; FVC: Forced vital capacity; ICD-10-CM: International Classification 
of Diseases 10 Clinical Modification; ICS: Inhaled corticosteroid; LABA: Longacting beta-agonists; LTRA: Leukotriene receptor antagonist; RR: Relative risk; SABA: Short-acting beta-agonists; SD: Standard deviation

\section{Acknowledgements}

The authors would like to thank Helena Goike (AstraZeneca employee) for her support throughout the conduct of this study.

\section{Authors' contribution}

All authors (KL, BS, KL, GT, GJ, MT, CJ) participated equally in the study conception, design, and statistical analysis planning. MT was responsible for statistical analyses, $\mathrm{KL}$ for the manuscript draft and finalization, and CJ for handling of data and the study database. Analysis and interpretation of data, and manuscript revisions were performed by all authors. All authors had access to complete study data, and had authority over manuscript preparation, approval of final version and the decision to submit for publication. $\mathrm{KL}$ is the guarantor.

\section{Funding}

AstraZeneca was the sponsor of this study.

\section{Availability of data and materials}

The datasets used and/or analyzed during the current study are available from the corresponding author on reasonable request.

\section{Ethics approval and consent to participate}

The study protocol was approved by the regional ethics committee in Uppsala, Sweden (reference number 2014/446).

\section{Consent for publication}

Not applicable.

\section{Competing interests}

All authors have completed the ICMJE uniform disclosure form at www.icmje.org/ coi_disclosure.pdf (available on request from the corresponding author) and declare: K. Larsson has received honoraria for participation in advisory board meetings and lectures from AstraZeneca, Boehringer Ingelheim, Novartis, Chiesi, Orion, TEVA and Takeda. B. Ställberg has served in advisory board meetings, development of educational presentations and for lectures from AstraZeneca, Novartis, Boehringer Ingelheim, Meda and TEVA. K. Lisspers has received speaking fees from AstraZeneca, Novartis, TEVA and Meda. C. Janson has received honoraria for lectures from AstraZeneca, TEVA and Novartis. G. Telg is a full-time employee of the sponsor, AstraZeneca. G. Johansson and M. Thuresson have no conflicts to report.

\section{Publisher's Note}

Springer Nature remains neutral with regard to jurisdictional claims in published maps and institutional affiliations.

\section{Author details}

'Work Environment Toxicology, The National Institute of Environmental Medicine, Karolinska Institutet, Stockholm, Sweden. ${ }^{2}$ Department of Public Health and Caring Sciences, Family Medicine and Preventive Medicine, Uppsala University, Uppsala, Sweden. ${ }^{3}$ AstraZeneca Nordic-Baltic, Södertälje, Sweden. ${ }^{4}$ Statisticon AB, Uppsala, Sweden. ${ }^{5}$ Department of Medical Sciences, Respiratory, Allergy and Sleep Research, Uppsala University, Uppsala, Sweden.

Received: 18 October 2017 Accepted: 8 January 2018

Published online: 18 January 2018

\section{References}

1. ToT SJ, Zhu J, Feldman L, Dell SD, Lougheed MD, et al. Asthma deaths in a large provincial health system a 10-year population-based study. Ann Am Thor Soc. 2014;11:1210-7.

2. Global Initiative for Asthma. Global strategy for asthma management and prevention.2016 wwwginasthmaorg.

3. Partridge MR, van der Molen T, Myrseth SE, Busse WW. Attitudes and actions of asthma patients on regular maintenance therapy: the INSPIRE study. BMC Pulm Med. 2006:6:13.

4. Rabe KF, Vermeire PA, Soriano JB, Maier WC. Clinical management of asthma in 1999: the asthma insights and reality in Europe (AIRE) study. Eur Respir J. 2000;16(5):802-7.
5. Custovic A, Johnston SL, Pavord I, Gaga M, Fabbri L, Bel EH, et al. EAACl position statement on asthma exacerbations and severe asthma. Allergy. 2013;68(12):1520-31.

6. Bardelas J, Figliomeni M, Kianifard F, Meng X. A 26-week, randomized, double-blind, placebo-controlled, multicenter study to evaluate the effect of omalizumab on asthma control in patients with persistent allergic asthma. J Asthma. 2012:49(2):144-52

7. Bousquet J, Siergiejko Z, Swiebocka E, Humbert M, Rabe KF, Smith N, et al. Persistency of response to omalizumab therapy in severe allergic (lgEmediated) asthma. Allergy. 2011;66(5):671-8

8. Chung KF, Wenzel SE, Brozek JL, Bush A, Castro M, Sterk PJ, et al. International ERS/ATS guidelines on definition, evaluation and treatment of severe asthma. Eur Respir J. 2014:43(2):343-73.

9. van Nooten F, Stern S, Braunstahl GJ, Thompson C, Groot M, Brown RE. Cost-effectiveness of omalizumab for uncontrolled allergic asthma in the Netherlands. J Med Econ. 2013;16(3):342-8.

10. Chanez P, Wenzel SE, Anderson GP, Anto JM, Bel EH, Boulet LP, et al. Severe asthma in adults: what are the important questions? J Allergy Clin Immunol. 2007;119(6):1337-48

11. von Bulow A, Kriegbaum M, Backer $V$, Porsbjerg $C$. The prevalence of severe asthma and low asthma control among Danish adults. J Allergy Clin Immunol Pract. 2014:2(6):759-67.

12. Makela MJ, Backer V, Hedegaard M, Larsson K. Adherence to inhaled therapies, health outcomes and costs in patients with asthma and COPD. Respir Med. 2013;107(10):1481-90.

13. Bjerg A, Ekerljung L, Middelveld R, Dahlen SE, Forsberg B, Franklin K, et al. Increased prevalence of symptoms of rhinitis but not of asthma between 1990 and 2008 in Swedish adults: comparisons of the ECRHS and GA(2)LEN surveys. PLoS One. 2011;6(2):e16082.

14. Martinell M, Stalhammar J, Hallqvist J. Automated data extraction-a feasible way to construct patient registers of primary care utilization. Ups J Med Sci. 2012;117(1):52-6.

15. Bel EH, Sousa A, Fleming L, Bush A, Chung KF, Versnel J, et al. Diagnosis and definition of severe refractory asthma: an international consensus statement from the innovative medicine initiative (IMI). Thorax. 2011:66(10):910-7.

16. O'Byrne PM, Naji N, Gauvreau GM. Severe asthma: future treatments. Clin Exp Allergy. 2012:42(5):706-11.

17. Hekking PP, Wener RR, Amelink M, Zwinderman AH, Bouvy ML, Bel EH. The prevalence of severe refractory asthma. J Allergy Clin Immunol. 2015;135(4): 896-902.

18. Gibson PG, McDonald VM. Asthma-COPD overlap 2015: now we are six Thorax. 2015:70(7):683-91.

19. Kostikas K, Clemens A, Patalano F. The asthma-COPD overlap syndrome: do we really need another syndrome in the already complex matrix of airway disease? Int J Chron Obstruct Pulmon Dis. 2016;11:1297-306.

20. Larsson K, Menzies-Gow A, Panettieri R. Severe asthma: challenges and precision approaches to therapy. Pulm Ther. 2016; https://doi.org/10.1007/ s41030-016-0022-2.

21. Kew KM, Seniukovich A. Inhaled steroids and risk of pneumonia for chronic obstructive pulmonary disease. Cochrane Database Syst Rev. 2014;3: CD010115.

22. Janson C, Larsson $K$, Lisspers KH, Stallberg B, Stratelis G, Goike $H$, et al. Pneumonia and pneumonia related mortality in patients with COPD treated with fixed combinations of inhaled corticosteroid and long acting beta2 agonist: observational matched cohort study (PATHOS). BMJ. 2013:346:f3306.

23. Suissa S, Patenaude V, Lapi F, Ernst P. Inhaled corticosteroids in COPD and the risk of serious pneumonia. Thorax. 2013:68(11):1029-36.

24. O'Byrne PM, Pedersen S, Carlsson LG, Radner F, Thoren A, Peterson S, et al. Risks of pneumonia in patients with asthma taking inhaled corticosteroids. Am J Respir Crit Care Med. 2011;183(5):589-95.

25. McKeever T, Harrison TW, Hubbard R, Shaw D. Inhaled corticosteroids and the risk of pneumonia in people with asthma: a case-control study. Chest. 2013;144(6):1788-94

26. Bender BG, Pedan A, Varasteh LT. Adherence and persistence with fluticasone propionate/salmeterol combination therapy. J Allergy Clin Immunol. 2006:118(4):899-904

27. Broder MS, Chang EY, Kamath T, Sapra S. Poor disease control among insured users of high-dose combination therapy for asthma. Allergy Asthma Proc. 2010;31(1):60-7. 